\title{
Bile Acid Signaling Is Involved in the Neurological Decline in a Murine Model of Acute Liver Failure
}

Matthew McMillin, ${ }^{* \dagger \dagger}$ Gabriel Frampton, ${ }^{* \dagger \dagger}$ Matthew Quinn, ${ }^{* \S}$ Samir Ashfaq, ${ }^{\dagger}$ Mario de los Santos, III, Stephanie Grant, ${ }^{* \dagger \ddagger}$ and Sharon DeMorrow ${ }^{\star}+\ddagger$

From the Department of Internal Medicine, * Texas A\&M Health Science Center, College of Medicine, Temple, Texas; the Digestive Disease Research Center, ${ }^{\dagger}$ Baylor Scott \& White Health, Temple, Texas; the Central Texas Veterans Healthcare System, ${ }^{\ddagger}$ Temple, Texas; and the Laboratory of Signal Transduction, ${ }^{\S}$ National Institute of Environmental Health Sciences, National Institutes of Health, Department of Health and Human Services, Research Triangle Park, North Carolina

Accepted for publication October 9, 2015.

Address correspondence to Sharon DeMorrow, Ph.D., Department of Internal Medicine, Baylor Scott \& White Health, Texas A\&M Health Science Center, and Central Texas Veterans Health Care System, $1901 \mathrm{~S} 1 \mathrm{st}$ St, Bldg 205, Temple, TX 76504. E-mail: demorrow@ medicine.tamhsc.edu.

\begin{abstract}
Hepatic encephalopathy is a serious neurological complication of liver failure. Serum bile acids are elevated after liver damage and may disrupt the blood-brain barrier and enter the brain. Our aim was to assess the role of serum bile acids in the neurological complications after acute liver failure. $\mathrm{C} 57 \mathrm{BL} / 6$ or cytochrome p450 7A1 knockout $\left(\mathrm{Cyp}_{7 \mathrm{~A}}{ }^{-/-}\right)$mice were fed a control, cholestyramine-containing, or bile acid-containing diet before azoxymethane (AOM)-induced acute liver failure. In parallel, mice were given an intracerebroventricular infusion of farnesoid $X$ receptor (FXR) Vivo-morpholino before AOM injection. Liver damage, neurological decline, and molecular analyses of bile acid signaling were performed. Total bile acid levels were increased in the cortex of AOM-treated mice. Reducing serum bile acids via cholestyramine feeding or using Cyp $7 A 1^{-/-}$mice reduced bile acid levels and delayed AOMinduced neurological decline, whereas cholic acid or deoxycholic acid feeding worsened AOM-induced neurological decline. The expression of bile acid signaling machinery apical sodium-dependent bile acid transporter, FXR, and small heterodimer partner increased in the frontal cortex, and blocking FXR signaling delayed $\mathrm{AOM}$-induced neurological decline. In conclusion, circulating bile acids may play a pathological role during hepatic encephalopathy, although precisely how they dysregulate normal brain function is unknown. Strategies to minimize serum bile acid concentrations may reduce the severity of neurological complications associated with liver failure. (Am J Pathol 2016, 186: 312-323; http:// dx.doi.org/10.1016/j.ajpath.2015.10.005)
\end{abstract}

Hepatic encephalopathy (HE) is a serious neuropsychiatric complication of both acute liver failure and chronic liver diseases, with the potential to affect health-related quality of life, clinical management strategies, priority for liver transplantation, and patient survival. ${ }^{1} \mathrm{HE}$ resulting from acute liver failure causes altered mental states and cognitive disruptions that can progress to coma in hours or days. ${ }^{2}$ Associated with the neuropsychiatric decline observed in $\mathrm{HE}$ are cerebral edema, neuroinflammation, increased intracranial pressure, and brain herniation. Therapies designed to detoxify the blood are often successful in alleviating the symptoms of mild or minimal HE. ${ }^{3}$ However, the treatment options for patients with more severe $\mathrm{HE}$ are limited, with liver transplantation being one of the only options that significantly enhances the chances of survival for these patients. ${ }^{4}$

Bile acids are synthesized in the liver from cholesterol primarily via a mechanism involving the enzyme cytochrome p450 7A1 (Cyp7A1) and can be conjugated, mainly

Supported by an NIH R01 award DK082435, an NIH K01 award DK078532, a Scott \& White Intramural grant award 050339, and a US Department of Veterans Affairs Biomedical Laboratory Research and Development Service VA merit award BX002638-01 (all to S.D.).

The contents do not represent the views of the US Department of Veterans Affairs of the US Government.

Disclosures: None declared. 
to glycine or taurine, before being released into the bile to aid in digestion. Conjugated bile acids, which carry a negative charge at physiological $\mathrm{pH}$, require carriermediated transport to cross membranes. ${ }^{5}$ The uptake of bile acids can occur via one of several transporters, including the apical sodium-dependent bile acid transporter (ASBT). ${ }^{6}$ Once bile acids gain entry into the cell, they can exert their effects through several nuclear receptors, including farnesoid X receptor (FXR). ${ }^{7}$ Activation of FXR results in the up-regulation of the cofactor small heterodimer partner (SHP), which works in concert with FXR to affect change in target gene expression. ${ }^{8}$

Under normal physiological conditions, $95 \%$ of the bile acid pool is reabsorbed from the intestine and transported to the liver, which constitutes an inhibitory feedback control on bile acid synthesis. During liver damage, there is increased accumulation of bile acids in the liver and a spillover of bile acids into the systemic circulation. These elevated serum bile acid levels have been associated with hepatotoxicity, ${ }^{9,10}$ hepatic fibrosis, ${ }^{11}$ pruritus, ${ }^{12}$ cardiomyopathy, ${ }^{13}$ and vasodilation. ${ }^{14}$ However, the role of bile acids in HE because of acute liver failure is unclear. Therefore, the aims of this study were to assess the effects of increased serum bile acid levels on the neurological decline associated with acute liver failure and to assess the possible mechanism by which this may occur.

\section{Materials and Methods}

\section{Reagents}

All chemicals were purchased from Sigma-Aldrich (St. Louis, MO) unless otherwise noted, and were of the highest grade available. Cholyl-lysyl fluorescein (CLF; a fluorescent bile acid derivative) was purchased from BD Biosciences (San Jose, CA). The FXR-specific antibody was purchased from Abcam (Cambridge, MA), and SHPspecific antibody was purchased from Santa Cruz Biotechnology (Dallas, TX). The antibody against ASBT was a kind gift from Dr. Paul Dawson (Wake Forest University Health Sciences, Winston Salem, NC). ${ }^{15}$ The primers for FXR (catalog number PPM24915A), ASBT (catalog number PPM24983A), Shp (catalog number PPM41772A), and glyceraldehyde-3-phosphate dehydrogenase (catalog number PPM02946E) were purchased from Qiagen, SABiosciences (Frederick, MD). Vivo-morpholino sequences were purchased from Gene Tools (Philomath, OR). Custom-made rodent diets were purchased from Dyets Inc. (Bethlehem, PA) and were made on the base diet formulation AIN-93G. Breeding pairs of Cyp7Al heterozygous knockout mice were a kind gift from Dr. Sandra Erickson (University of California, San Francisco, CA). ${ }^{16}$ Cyp $7 A 1$ heterozygous knockout mice were bred on a $C 57 B / / 6$ background to establish a breeding colony. Homozygous Cyp7Al knockout mice $\left(C y p 7 A 1^{-1-}\right)$ and wild-type (WT) littermates were used in all experiments.

\section{In Vivo Model of Acute Liver Failure}

Mouse in vivo experiments were performed using male C57Bl/6 mice (25 to $30 \mathrm{~g}$; Charles River Laboratories, Wilmington, MA) or in male Cyp $7 A 1^{-1-}$ or WT littermate controls with approval from Baylor Scott \& White Health (Temple, TX) and Texas A\&M Health Science Center (Temple, TX) Institutional Animal Care and Use Committees. Mice received a single i.p. injection of $100 \mu \mathrm{g} / \mathrm{g}$ of azoxymethane (AOM) to induce acute liver failure and $\mathrm{HE}$. After injection, mice were placed on heating pads set to $37^{\circ} \mathrm{C}$ to ensure they remained normothermic. Hydrogel and rodent chow were placed on cage floors to ensure easy access to food and hydration. After 12 hours and every 4 hours thereafter, mice were injected s.c. with 5\% dextrose in $250 \mu \mathrm{L}$ saline to ensure euglycemia and hydration. After injection, mice were monitored at least every 2 hours (starting at 8 hours after AOM injection) for body temperature, weight, and neurological decline. ${ }^{17-19}$ Once neurological decline developed, mice were continuously monitored, with formal assessments of temperature, body weight, and neurological decline performed each hour. Neurological decline was assessed by measuring the pinna reflex, corneal reflex, tail flexion, escape response, righting reflex, and ataxia, as described previously ${ }^{17-19}$ by an investigator blinded to the treatments (M.M. or S.G.). The summation of these six reflexes gives a neurological score between 0 and 12 .

Tissue was collected before neurological symptoms (preneurological), when minor ataxia and weakened reflexes were present (minor neurological), when major ataxia and deficits in reflexes were evident (major neurological), or at coma, where there was loss of righting and corneal reflexes, as described previously. ${ }^{17,18}$ Cerebral edema was assessed in all mice using the wet/dry weight method described by Baskaya et al. ${ }^{20}$ Water content was expressed as a percentage of brain weight, calculated as follows: ([wet weight - dry weight $] /$ wet weight $) \times 100 \%$. Increased brain water content of $1 \%$ to $2 \%$ in mice is indicative of cerebral edema and is characterized by increased intracranial pressure. ${ }^{21,22}$

\section{Pharmacological Manipulations in AOM Mice}

In a subset of experiments, mice were fed a diet containing cholestyramine $(2 \%)$, cholic acid $(0.3 \%)$, deoxycholic acid (DCA; $0.3 \%$ ), and ursodeoxycholic acid (UDCA; 3\%) or the control diet AIN-93G for 3 days before the injection of AOM. The concentrations of bile acids in these diets have been previously shown to have no significant hepatotoxic effects. $^{23}$ Specific inhibition of FXR expression in the frontal cortex was achieved by directly infusing an FXRspecific Vivo-morpholino sequence (5'-CTGAAACTGCATCACCATCCTTAGC- $\left.3^{\prime}\right)$ or a mismatched control (5'CTCAAAGTGGATCACCATCGTTACC-3') using brain infusion kits coupled to s.c. implanted minipumps (Alzet, Cupertino, CA) at $1 \mathrm{mg} / \mathrm{kg}$ per day for 3 days before AOM injection at coordinates AP 2.0, ML -2.0 , and DV -2.5 . 


\section{Assessment of Bile Acid Content in the Brain}

At the time when minor neurological symptoms were present, the mice were anesthetized and transcardially perfused with ice-cold saline to remove the blood from the brain. Cortex homogenates were prepared by calculating the wet weight of brain tissue with subsequent homogenization in $100 \mathrm{mg} / \mathrm{mL}$ in ultrapure water using a Miltenyi Biotec gentleMACS Dissociator (San Diego, CA). Homogenates were spun down for 5 minutes at $16,100 \times g$, and supernatants were collected. Total bile acid content was assessed in homogenates of the frontal cortex following the manufacturer's instructions (Diazyme Laboratories, Poway, CA). To assess the performance of this kit, brain homogenates were supplemented with DCA to a final concentration of $10 \mu \mathrm{mol} / \mathrm{L}$ after homogenization in ultrapure water, as outlined above. This concentration was chosen as approximately equivalent to the total bile acid concentration reached in the serum. The original lysate and the DCAsupplemented lysate were subsequently used for the total bile acid assay, and the percentage of bile acid recovery was assessed by the following formula: ([DCA-supplemented lysate bile acid concentration - control lysate bile acid concentration $] / 10 \mu \mathrm{mol} / \mathrm{L}) \times 100 \%$.

In parallel, mice were given a single tail vein injection of the fluorescent bile acid derivative CLF (BD Biosciences) 1 hour before the injection of AOM. CLF exhibits aspects of transporter-mediated transport like endogenous bile acids and shows similar brain penetrance to tritiated taurocholate in models of chronic liver damage in our hands (data not shown). ${ }^{24,25}$ Once again, at the time when minor neurological symptoms were evident, mice were anesthetized and transcardially perfused with ice-cold saline to remove the blood from the brain and the amount of fluorescence in homogenates of the frontal cortex was assessed as previously described. ${ }^{26}$

\section{Liver Biochemistry}

Plasma alanine aminotransferase (ALT) was assessed using commercially available kits. ALT measurement was performed using a fluorimetric activity assay (Sigma-Aldrich), according to manufacturer's instructions.

\section{Molecular Analysis}

Expression and subcellular localization of the described target genes were assessed by real-time PCR, ${ }^{27}$ immunoblotting, ${ }^{28}$ immunohistochemistry, ${ }^{29}$ or immunofluorescence, ${ }^{28}$ as previously described.

\section{Real-Time PCR}

RNA was extracted from tissues or cells, and real-time PCR was performed, as previously described, ${ }^{30}$ using commercially available primers designed against mouse ASBT,
FXR, SHP, and glyceraldehyde-3-phosphate dehydrogenase (SABiosciences). A $\Delta \Delta \mathrm{CT}$ analysis was performed using vehicle-treated tissue or untreated primary neurons as controls for subsequent experiments. ${ }^{31,32}$ Data for all experiments were expressed as mean relative mRNA levels \pm SEM.

\section{Immunoblotting}

SDS-PAGE gels $(10 \% \mathrm{v} / \mathrm{v})$ were loaded with 10 to $20 \mu \mathrm{g}$ of protein diluted in Laemmli buffer per tissue sample. Specific antibodies against ASBT, FXR, SHP, and $\beta$-actin were used. All imaging was performed on an Odyssey 9120 Infrared Imaging System (LI-COR, Lincoln, NE). Data are expressed as fold change in fluorescent band intensity of target antibody divided by $\beta$-actin, which is used as a loading control. The values of vehicle or control groups were used as a baseline and set to a relative protein expression value of 1 . All treatment groups were expressed as changes of fluorescent band intensity of target antibody to $\beta$-actin relative to vehicle or control groups. Band intensity quantifications were performed using ImageJ software version 10.2 (NIH, Bethesda, MD; http://imagej.nih.gov/ij). Data for all experiments were expressed as mean relative protein \pm SEM.

\section{Histology, Immunohistochemistry, and Immunofluorescence}

Paraffin-embedded livers were cut into sections ( $3 \mu \mathrm{m}$ thick) and mounted onto glass slides where they were used for hematoxylin and eosin staining. ${ }^{33}$ For brain immunohistochemistry and immunofluorescence, free-floating sections (30 $\mu \mathrm{m}$ thick) were selected and immunoreactivity was assessed using specific antibodies for ASBT, FXR, and the neuronal marker NeuN. The sections were viewed using an Olympus BX40 microscope with an Olympus DP25 imaging system (Olympus, Center Valley, PA) or a Leica TCS SP5-X inverted confocal microscope (Leica Microsystems, Buffalo Grove, IL).

\section{In Vitro Studies}

Primary cortical neurons were isolated as previously described, ${ }^{17}$ allowed to differentiate, and treated with 10 $\mu \mathrm{mol} / \mathrm{L} \mathrm{DCA}$ in the presence or absence of $10 \mu \mathrm{mol} / \mathrm{L}$ FXR antagonist guggulsterone for 24 hours. FXR nuclear translocation was assessed by immunofluorescence microscopy, as described previously, ${ }^{30}$ and neurons were counterstained with the nuclear stain DAPI. In parallel, SHP expression was assessed by real-time PCR, as described previously. ${ }^{27}$

\section{Statistical Analysis}

All statistical analyses were performed using GraphPad Prism software version 5.04 (GraphPad Software, La Jolla, 

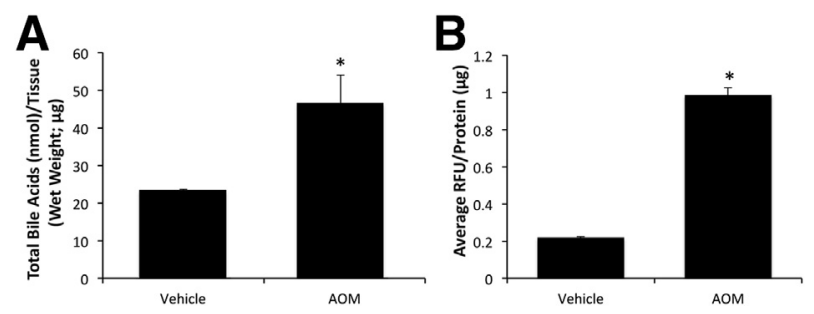

Figure 1 Bile acids are elevated in the brain after liver failure. A: Total bile acid quantification in the frontal cortex of mice treated with vehicle or azoxymethane (AOM). B: Relative fluorescence in the cortex of vehicle or AOM-treated mice injected with cholyl-lysyl-fluorescein (CLF). Data are expressed as means $\pm \mathrm{SEM}$ ( $\mathbf{A}$ and $\mathbf{B}$ ). $n=4$ (A and $\mathbf{B}$ ). ${ }^{*} P<0.05$ versus vehicle-injected mice. $\mathrm{RFU}$, relative fluorescence units.

CA). For data that passed normality tests, significance was established using the $t$-test when differences between two groups were analyzed, and analysis of variance when differences between three or more groups were compared, followed by the appropriate post hoc test. If tests for normality failed, two groups were compared with a $U$ test or a Kruskal-Wallis ranked analysis when more than two groups were analyzed. Results were expressed as means \pm SEM. Differences were considered significant when $P<0.05$.

\section{Results}

Total Bile Acid Concentrations Are Elevated in the Brain Following Acute Liver Failure

To determine whether the increased serum bile acids reported after liver failure result in increased bile acid content in the brain, total bile acid assays were performed on cortical tissue from vehicle and AOM-treated mice. Because this assay had been validated in liver tissue, but not brain tissue, a spiked brain homogenate assay was performed. DCA supplement $(10 \mu \mathrm{mol} / \mathrm{L})$ to brain homogenates was recovered at $105.81 \% \pm 19.93 \%$, indicating that the assay could be used reliably for brain tissue at the concentrations measured. Using this assay, there was a significant increase in the amount of total bile acids in the cortex after AOM treatment compared with control (Figure 1A). Furthermore, tail vein injection of a fluorescent bile acid derivative CLF, before the injection of AOM, resulted in an increased amount of fluorescence detected in cortical tissue after AOM injection (Figure 1B). Taken together, these data suggest that the increased total bile acid content in the brain during acute liver failure could be derived from the circulation.

\section{Reducing Circulating Bile Acids Confers Protection against Acute Liver Failure-Induced Neurological Impairment}

To assess the effects of modulating the bile acid pool, mice were fed a diet enriched with the bile acid sequestrant cholestyramine for 3 days, a modality that significantly reduced the total bile acid content in the serum (Figure 2A). Mice that were fed a cholestyramine-supplemented diet before treatment with AOM displayed delayed neurological decline (Figure 2B) and increased time taken to reach coma (Figure 2C) compared with control diet-fed mice. The percentage of brain water, a measure of cerebral edema, at

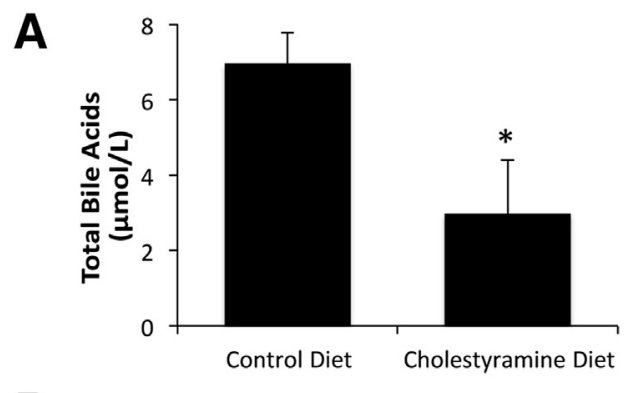

B
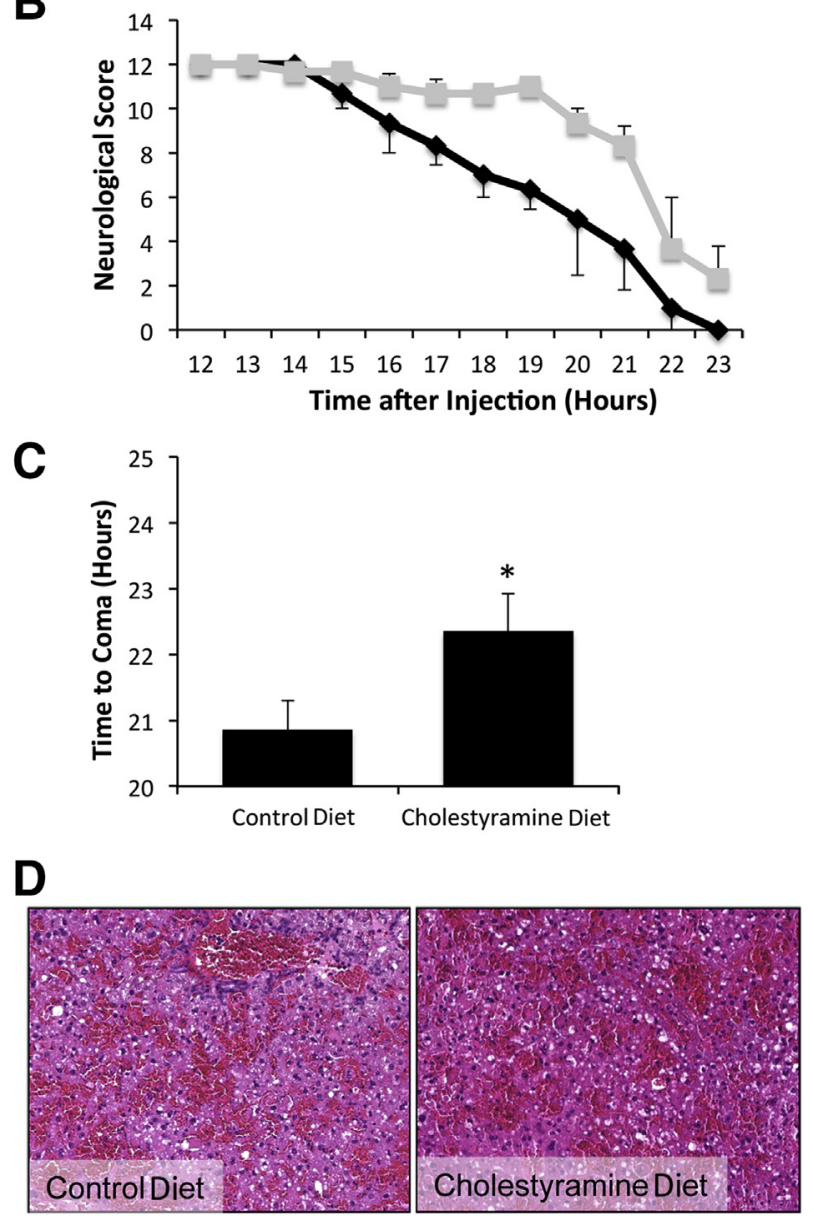

Figure 2 Reduction of circulating bile acids is protective against acute liver failure-induced neurological impairment. A: Serum bile acid concentrations in mice that ingest a control or cholestyramine-supplemented diet. B: Neurological score of azoxymethane (AOM)-treated mice that consume control (black) or cholestyramine-supplemented (gray) diet. C: Time to coma in hours for A0M-treated mice that were fed either control or cholestyramine diets. D: Hematoxylin and eosin staining of AOM-treated mice fed with control or cholestyramine diets. ${ }^{\star} P<0.05$ versus control diet mice. $n=5(\mathbf{A}-\mathbf{C})$. 
Table 1 Serum Liver Enzymes for Treated Mice

\begin{tabular}{|c|c|}
\hline Variable & $\operatorname{ALT}(\mathrm{U} / \mathrm{L})$, means $\pm \mathrm{SEM}$ \\
\hline Vehicle & $18.09 \pm 3.82$ \\
\hline $\mathrm{AOM}$ & $188.62 \pm 20.51^{*}$ \\
\hline Cholestyramine & $22.68 \pm 0.98$ \\
\hline Cholestyramine $+\mathrm{AOM}$ & $287.34 \pm 20.54^{*}$ \\
\hline Cyp $7 A 1^{-/-}+$vehicle & $16.74 \pm 1.95$ \\
\hline Cyp7A1 ${ }^{-/-}+\mathrm{AOM}$ & $497.05 \pm 23.34^{*}$ \\
\hline Control diet & $28.45 \pm 9.21$ \\
\hline Control diet $+\mathrm{AOM}$ & $104.67 \pm 0.77^{*}$ \\
\hline Cholic acid diet & $31.91 \pm 15.61$ \\
\hline Cholic acid diet + AOM & $149.95 \pm 4.63^{*}$ \\
\hline DCA diet & $80.41 \pm 6.84$ \\
\hline $\mathrm{DCA}$ diet $+\mathrm{AOM}$ & $176.52 \pm 15.50^{*}$ \\
\hline UDCA diet & $27.17 \pm 0.58$ \\
\hline UDCA diet + AOM & $99.04 \pm 4.76^{*}$ \\
\hline FXR mismatch VM & $17.03 \pm 0.48$ \\
\hline FXR mismatch $\mathrm{VM}+\mathrm{AOM}$ & $167.51 \pm 33.64^{*}$ \\
\hline FXR VM & $4.03 \pm 3.81$ \\
\hline FXR VM + AOM & $183.88 \pm 11.45^{*}$ \\
\hline
\end{tabular}

The normal range for ALT is from 17 to $77 \mathrm{U} / \mathrm{L}$.

${ }^{*} P<0.05$ versus vehicle.

ALT, alanine aminotransferase; A0M, azoxymethane; Cyp7A1, cytochrome p450 7A1; DCA, deoxycholic acid; FXR, farnesoid $X$ receptor; UDCA, ursodeoxycholic acid; VM, Vivo-morpholino.

coma in control-fed AOM-treated mice was significantly reduced in cholestyramine-fed mice treated with AOM $(82.52 \% \pm 0.59 \%$ and $80.53 \% \pm 0.19 \%$, respectively; $P=0.0315)$. Cholestyramine treatment did not have a significant effect on the liver damage, as shown by hematoxylin and eosin staining (Figure 2D) or serum chemistry (Table 1), suggesting that reducing bile acid concentrations is neuroprotective, rather than hepatoprotective.

In parallel, Cyp $7 A 1^{-1-}$ mice were used to further assess the effects of an altered bile acid content on the neurological decline associated with acute liver failure. $C y p 7 A 1^{-1-}$ mice have previously been shown to have reduced fecal bile acid content and total bile acid pool, as well as reduced concentrations of DCA, lithocholic acid, and $\beta$-muricholic acid. ${ }^{16}$ Reduced circulating bile acid content was confirmed in $C y p 7 \mathrm{Al}^{-1-}$ compared with WT controls (Figure 3A). Treatment of $C y p 7 A 1^{-1-}$ mice with AOM demonstrated significantly delayed neurological decline (Figure 3B) and increased time to coma (Figure 3C) compared with WT mice. Once again, AOM injection caused similar levels of liver damage in $C y p 7 A 1^{-1-}$ and WT mice, as demonstrated by hematoxylin and eosin staining (Figure 3D) and serum ALT levels (Table 1), again confirming that modulating the bile acid content in this manner was predominantly neuroprotective, rather than hepatoprotective.

\section{Bile Acid Pool Enrichment Differentially Affects Neurological Decline Due to Acute Liver Failure}

To further assess the involvement of various bile acids in the neurological decline associated with acute liver failure, mice were fed a diet enriched with DCA, its corresponding primary bile acid, cholic acid, or the protective bile acid UDCA for 3 days before the injection of AOM. Feeding with either DCA or cholic acid worsened the neurological decline (Figure 4A) and significantly decreased the time taken to reach coma (Figure 4B), with DCA having the

\section{A}

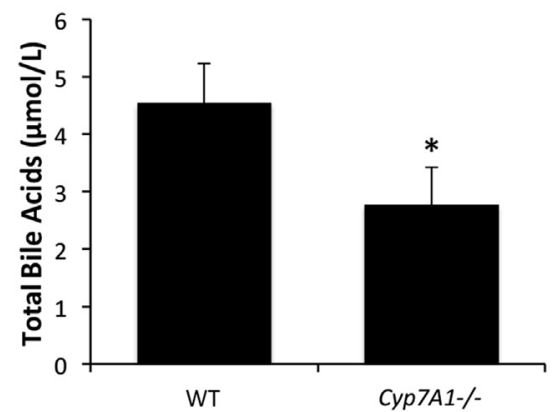

B

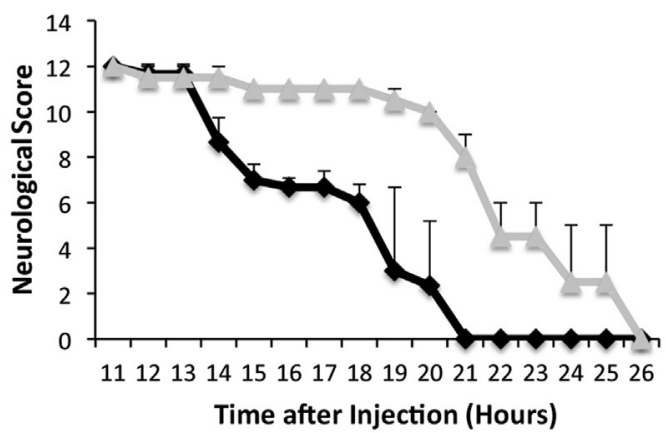

C

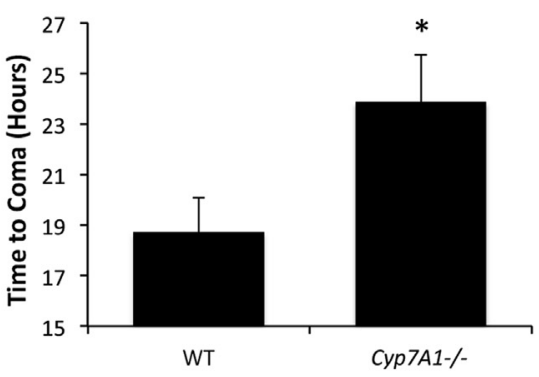

D

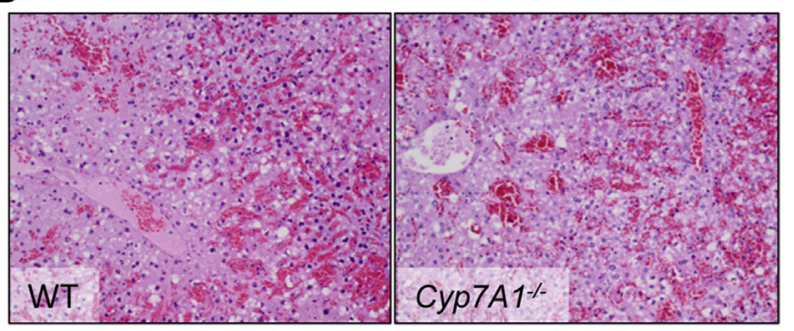

Figure 3 Knockout of cytochrome p450 7A1 (Cyp7a1) reduces circulating bile acids and neurological decline associated with acute liver failure. A: Wild-type (WT) and Cyp7a1 ${ }^{-/-}$mice serum bile acid levels. B: Neurological decline of azoxymethane (AOM)-treated WT (black) and Cyp7a1 ${ }^{-/}$(gray) mice. C: Time to coma in hours for AOM-treated WT and Cyp7a1 ${ }^{-/-}$mice. D: Representative hematoxylin and eosin staining in WT and $C y p 7 a 1^{-/-}$mice treated with AOM. ${ }^{\star} P<0.05$ versus WT mice. $n=5$ $(\mathbf{A}-\mathrm{C})$. 

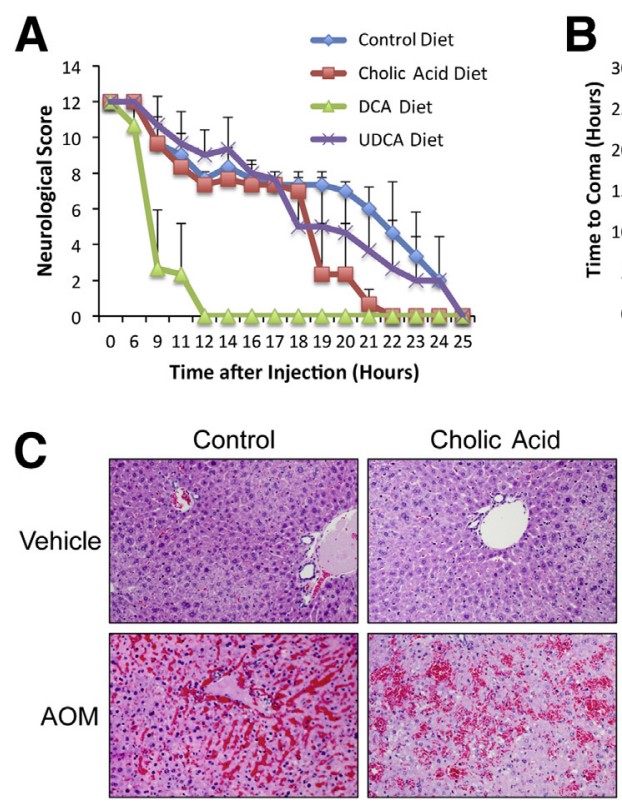

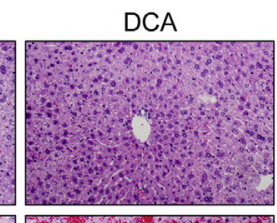

B
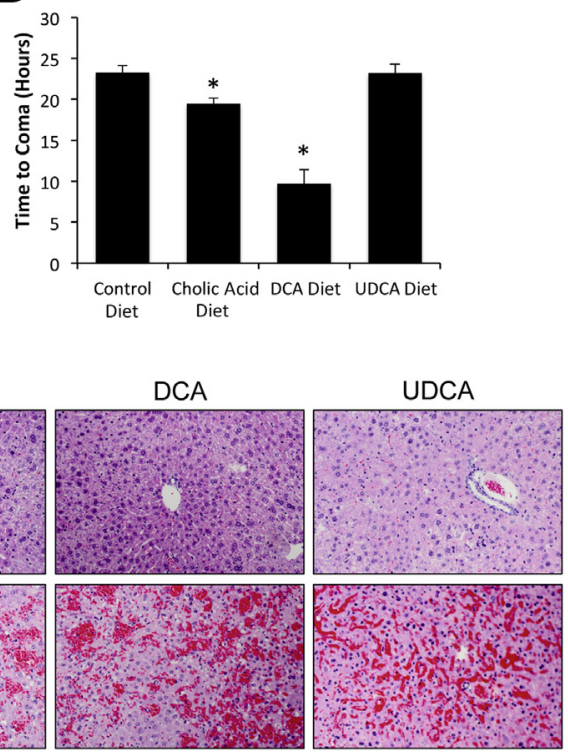

Figure 4 Enrichment of bile acid pools differentially affects the neurological decline associated with acute liver failure. A: Neurological decline in azoxymethane (AOM)-treated mice that were fed diets supplemented with cholic acid, deoxycholic acid (DCA), ursodeoxycholic acid (UDCA), or a control diet. B: Time to coma in hours for hepatic encephalopathy mice that consumed diets supplemented with cholic acid, DCA, UDCA, or a control diet. C: Hematoxylin and eosin histochemistry in control or AOM-treated mice that ingested diets supplemented with cholic acid, DCA, UDCA, or a control diet. ${ }^{*} P<0.05$ versus control diet-fed mice. $n=5$ (A and $\mathbf{B})$. greatest effect. Mice fed CA or DCA diets and administered AOM had a significant elevation of brain water compared with control diet mice treated with vehicle (CA: $81.72 \% \pm 0.28 \%, P=0.0012$; DCA: $80.78 \% \pm 0.30 \%$, $P=0.0328$; control: $79.65 \% \pm 0.32 \%$ ). UDCA-fed mice administered AOM had levels of cerebral edema comparable to control diet-fed mice administered vehicle (UDCA: $80.39 \% \pm 0.44 \%, P=0.2109$; control: $79.65 \% \pm 0.32 \%$ ). Interestingly, although there was no observable evidence of liver damage in any of the bile acid-treated mice before the injection of AOM (Figure 4C), there was a significant increase in ALT in the serum of DCA-fed mice, suggesting that the dramatic increase in the onset of neurological impairment in these mice may have been, in part, because of the presence of liver damage before the injection of AOM. However, the liver damage after AOM injection was comparable between treatment groups (Figure 4C and Table 1). UDCA feeding had no effect on the associated liver damage or neurological decline (Figure 4, A-C, and Table 1).

\section{FXR Signaling in the Brain is Up-Regulated Following Acute Liver Failure}

To dissect a possible mechanism by which bile acids may be exerting their effects, we assessed the expression of key components of the bile acid signaling pathway in the brain at stages of HE corresponding to the following: i) before the onset of neurological symptoms, ii) when minor neurological symptoms are present, iii) when major neurological impairment is observed, and iv) at the time of coma. These stages have been previously described in detail. ${ }^{17}$ Expression of the bile acid transporter ASBT, the bile acid nuclear receptor FXR, and its cofactor SHP was significantly increased after AOM injection in the frontal cortex before the onset of neurological symptoms compared with control tissue (Figure 5A and Supplemental Figure S1A), an effect that was observed through to major neurological decline. Interestingly, the expression of these key bile acid signaling components was dramatically decreased at the time of coma (Figure 5A and Supplemental Figure S1A). Expression of other bile acid transporters, such as hepatic sodium bile acid cotransporting polypeptide or organic anion transporting polypeptide, was either not detected or not changed (data not shown). By immunofluorescence, ASBT and FXR immunoreactivity was found to predominantly colocalize with the neuronal marker NeuN in a subset of neurons in the frontal cortex after AOM injection (Figure 5B). Interestingly, the AOM-induced increase in ASBT, FXR, and SHP expression was not observed in $C y p 7 A 1^{-1-}$ mice (Figure 5C and Supplemental Figure S1B). To demonstrate that this bile acid signaling pathway was functional in these neurons, primary cortical neurons were treated with DCA in the presence or absence of the FXR antagonist, guggulsterone, and functional markers of FXR activity were assessed. Specifically, under basal conditions, FXR immunoreactivity was found to be predominantly cytoplasmic, whereas after DCA treatment, FXR was found predominantly in the nucleus, an effect that could be blocked by pretreatment with the FXR antagonist guggulsterone (Figure 6A). Furthermore, DCA treatment of primary neurons resulted in an increase in SHP mRNA expression, a downstream target of FXR activation (Figure 6B), which again could be blocked by pretreatment with the FXR antagonist guggulsterone.

\section{Neural FXR Signaling Contributes to Neurological Decline Following Acute Liver Failure}

To determine whether FXR-mediated signaling was involved in the neurological decline associated with acute liver failure, an FXR-specific or mismatched control 
Vivo-morpholino sequence was infused directly into the frontal cortex for 3 days before the injection of AOM. This treatment regimen was used to dissect the specific effects of reduced FXR expression in the brain and was found to specifically decrease the expression of FXR in the frontal cortex adjacent to the injection site (Figure 7, A and B). No change in FXR expression was detected in the liver in these mice (data not shown). Pretreatment with the FXR-specific Vivo-morpholino sequence before AOM injection significantly delayed the neurological decline (Figure 7C) and increased the time to coma (Figure 7D) when compared with the mismatched control sequences. There was no liver damage because of the intracortical infusion of either Vivo-morpholino sequence, as assessed by hematoxylin and eosin staining (Figure 7E) and serum ALT levels (Table 1). Furthermore, the AOM injection caused a similar degree of liver damage after either Vivo-morpholino sequence (Figure 7E and Table 1), suggesting that down-regulating FXR expression in the frontal cortex is neuroprotective, but not hepatoprotective.

\section{Discussion}

HE is a serious neurological complication that arises after liver disease. ${ }^{34}$ Currently, HE has few effective treatments and thus the need to identify targets for potential therapeutics is important. Herein, we demonstrate the following: i) bile acid content in the frontal cortex is increased during acute liver failure, ii) strategies to modulate the bile acid content in the brain alter the neurological decline observed, and iii) FXR-mediated signaling contributes to the neurological impairment. Taken together, our data suggest that aberrant bile acid signaling in the brain may contribute to HE because of acute liver failure and that strategies to alter the bile acid content in the brain may alleviate the neurological consequences observed in acute liver failure.

\section{The AOM Model of Acute Liver Failure Is a Valid Model} of $\mathrm{HE}$

The AOM model of acute liver failure exhibits many of the pathophysiological characteristics of human HE because of acute liver failure. These features include the following: i) a clear pattern of neurological behaviors starting with the prodromal phase because of liver failure, where neurological symptoms are not yet evident (defined by us as the preneurological phase), followed by several distinct phases of neurological decline that rapidly progress to stupor or coma; ii) the presence of cerebral edema; and iii) high levels of ammonia in the blood and brain. In a comparative study, ${ }^{35}$

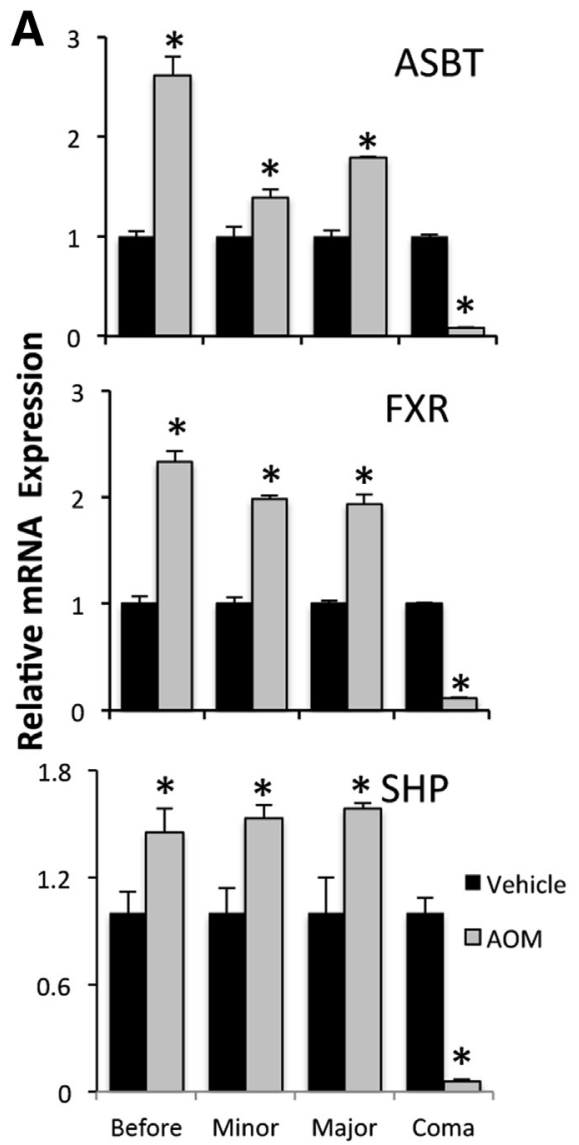

Stage of neurological decline

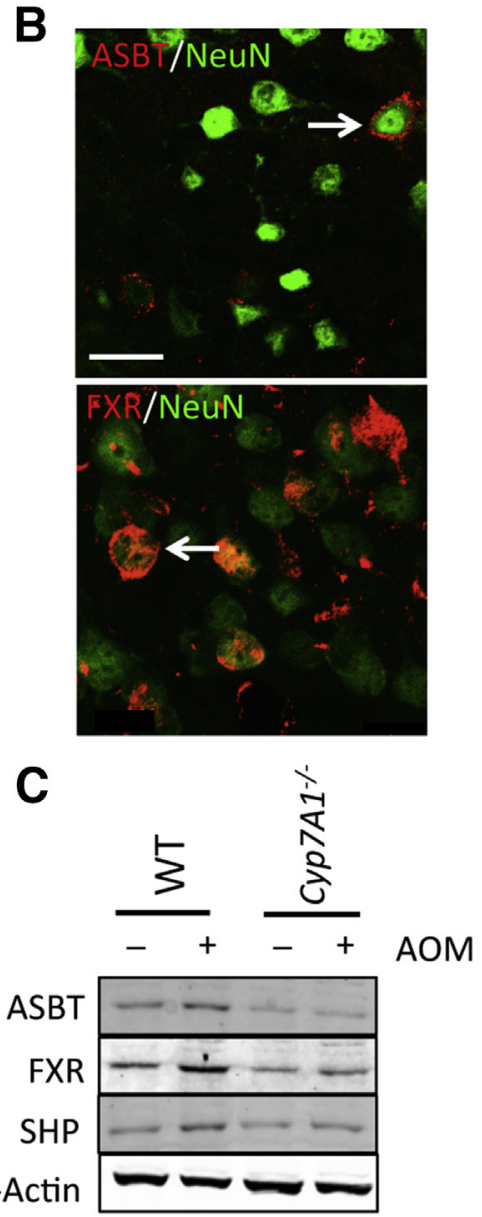

Figure 5 Farnesoid $X$ receptor (FXR) signaling is elevated in brain during acute liver failure. A: Apical sodium-dependent bile acid transporter (ASBT), FXR, and small heterodimer partner (SHP) cortical mRNA expression during the time course of neurological decline after azoxymethane (AOM) injection. B: Fluorescence immunoreactivity of ASBT and FXR (red) costained with neuronal marker NeuN (green) in the cortex. The arrows indicate colocalization of ASBT and NeuN immunoreactivity (top panel) and FXR and NeuN immunoreactivity (bottom panel). C: Immunoblots for ASBT, FXR, and SHP in wild-type (WT) and Cyp7a1-/- mice treated with control or AOM. $\beta$-Actin is used as a loading control. ${ }^{*} P<0.05$ versus vehicle-treated mice. $n=6$ (A). Scale bar $=25 \mu \mathrm{m}(\mathbf{B})$. 
A

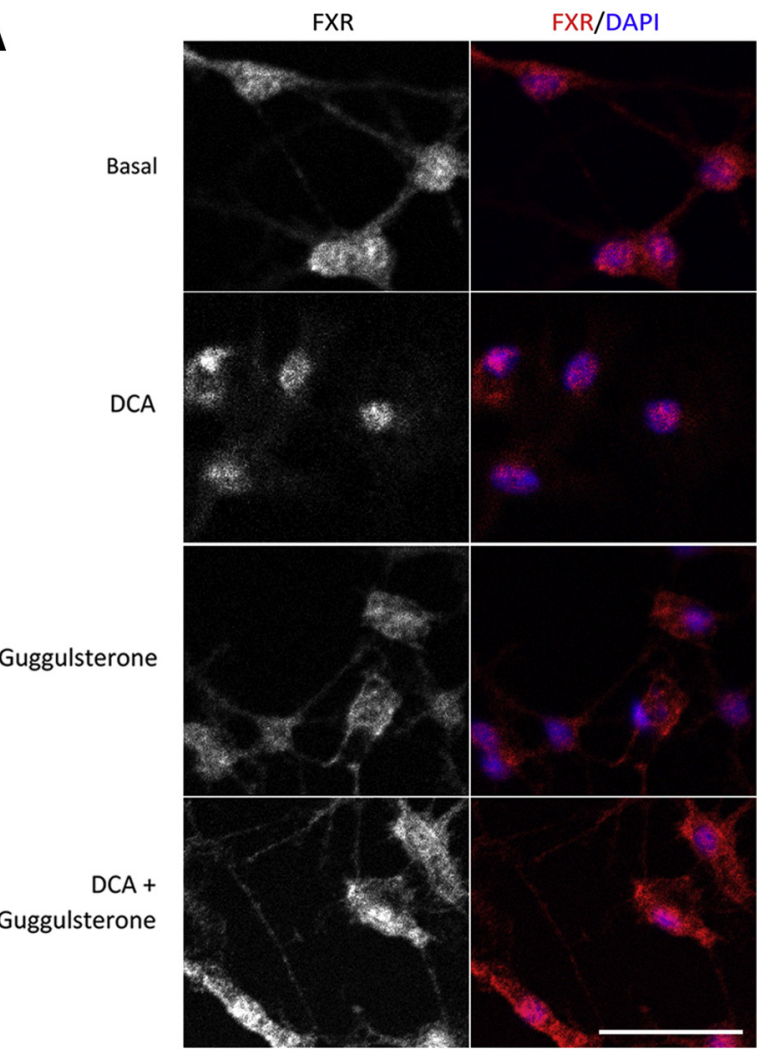

B

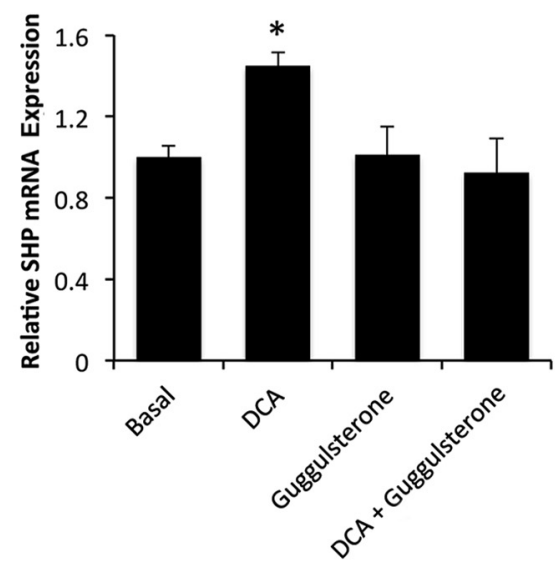

Figure 6 Primary neurons transduce farnesoid $X$ receptor (FXR) signaling in response to deoxycholic acid (DCA). A: Immunocytochemistry for FXR (red) of primary neurons treated with $10 \mu \mathrm{mol} / \mathrm{L} \mathrm{DCA}, 10 \mu \mathrm{mol} / \mathrm{L}$ guggulsterone, or both. DAPI (blue) is used as a nuclear marker. B: Relative small heterodimer partner (SHP) mRNA expression in primary neurons treated with $10 \mu \mathrm{mol} / \mathrm{L} \mathrm{DCA}, 10 \mu \mathrm{mol} / \mathrm{L}$ guggulsterone, or both. ${ }^{*} P<0.05$ versus basal primary neurons. $n=4$ (B). Scale bar $=25 \mu \mathrm{m}(\mathbf{A})$.

unlike other mouse models, the AOM model of HE was found to be the most reproducible, was reversible to a certain degree, and had a reasonable therapeutic window, with the neurological decline ultimately resulting in liverrelated death (similar to that observed in humans), as long as the body temperature and other physiological parameters are tightly controlled. Recently, it has been suggested that AOM is a flawed model of HE because AOM may be directly toxic on brain endothelial cells, thereby opening the blood-brain barrier (BBB). ${ }^{36}$ However, this study was purely an in vitro study using a coculture of brain endothelial cells and astrocytes in which the hepatotoxins were applied to the astrocyte layer rather than to the endothelial cells directly, as would be the case in an in vivo setting. Indeed, we have recently demonstrated that direct treatment of endothelial cells with AOM does not lead to increased monolayer permeability. ${ }^{37}$ Nguyen et $\mathrm{al}^{38}$ and our group have demonstrated that the BBB has increased permeability after AOM in vivo, but only at the later stages of encephalopathy, well beyond the onset of neurological symptoms. ${ }^{37}$ Conversely, others have shown that mice injected with AOM alone do not have increased BBB breakdown alone, but require a priming dose of lipopolysaccharide for the BBB permeability to be evident. ${ }^{39}$ Together, these in vivo reports do not support the idea that AOM is directly causing leakiness of the BBB; rather, it is a consequence of liver failure and the resulting complications that arise.

\section{Bile Acids Contribute to the Pathogenesis of HE}

Our current understanding of HE relies heavily on the idea that the buildup of ammonia in the bloodstream and brain is driving the neurological decline observed after acute and chronic liver failure. However, recent studies suggest that ammonia toxicity is not the only driving force in this condition. Indeed, current data suggest that there may be a synergism between ammonia and neuroinflammation, leading to the development of HE. ${ }^{40}$ Increased expression of the inflammatory chemokine ligand 2 has been attributed to AOM-induced HE progression and knockout of the IL-1 receptor, or the tumor necrosis factor receptor confers neuroprotection after AOM-induced HE. ${ }^{18,41}$ Data from the AOM model of acute liver failure indicate that ammonia concentrations are elevated only in the later stages of $\mathrm{HE}$, well beyond the onset of overt neurological impairment, ${ }^{42}$ indicating that other factors must be playing a role in the early stages of encephalopathy onset. The data presented herein suggest that serum bile acids may also be playing a role in the pathogenesis of HE.

It has long been acknowledged that serum bile acid levels increase as a result of liver damage because of the impaired reuptake mechanism in the liver, and increased bile acids in the circulation likely contribute to the peripheral adverse effects of liver damage, such as pruritus, ${ }^{12}$ cardiomyopathy, ${ }^{13}$ and vasodilation. ${ }^{14}$ Interestingly, the serum bile acid profile is altered in patients with cirrhosis, in whom there is a higher amount of primary bile acids, indicating a decreased bioconversion of primary to secondary bile acids, which may be attributable to alterations in the gut microflora. ${ }^{43}$ Herein, we provide evidence that serum bile acids contribute to the neurological consequences of acute liver failure as well. Our data indicate that there is an increase in total bile acid content in the frontal cortex of AOM-treated mice and that this increase is predominantly from the circulation. In support of our data, Bron et $\mathrm{al}^{44}$ demonstrated 

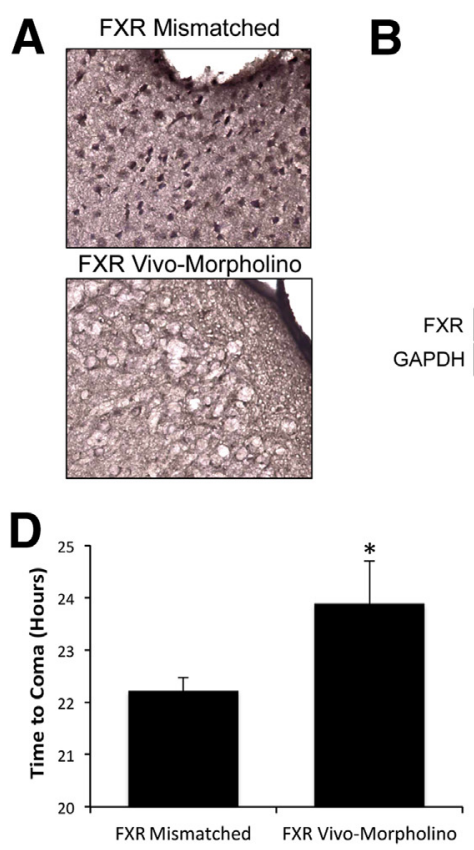

B
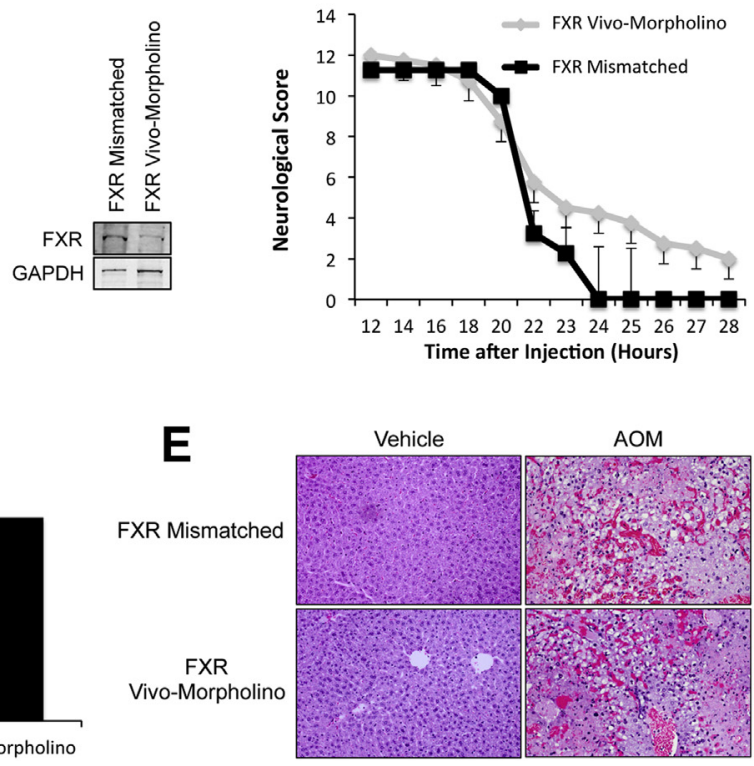

Figure 7 Neurological decline during acute liver failure is exacerbated via farnesoid $X$ receptor (FXR) signaling. A: FXR immunhohistochemisty in the cortex of mice after intracortical infusion with FXR-mismatched morpholino or FXR Vivomorpholino. B: Immunoblot against FXR with glyceraldehyde-3-phosphate dehydrogenase (GAPDH) used as a loading control in FXRmismatched morpholino or FXR Vivo-morpholino mice. C: Neurological decline in azoxymethane (AOM) mice infused with FXR-mismatched morpholino or FXR Vivo-morpholino. D: Time to coma in hours of AOM mice infused with FXR-mismatched morpholino or FXR Vivo-morpholino. E: Hematoxylin and eosin histochemistry in vehicle and AOM mice treated with FXR-mismatched morpholino or FXR Vivo-morpholino. ${ }^{*} P<0.05$ versus FXRmismatched morpholino mice. $n=5$ (C and $\mathbf{D})$. that patients who died from fulminant hepatic failure had small, but significant, amounts of bile acids in the cerebrospinal fluid that were absent from patients dying without evidence of liver disease. Furthermore, in bile duct-ligated rats, the bile acid profile in the brain was found to have a more toxic composition than that of controls. ${ }^{45}$ Specifically, $87.4 \%$ of the total bile acids detected were found to be in the form of lithocholic acid, a toxic hydrophobic bile acid, which was absent in control animals. ${ }^{45}$ This study primarily focused on bile acid levels and associated molecular changes in the frontal cortex because this region is significantly affected during $\mathrm{HE},{ }^{46-48}$ although other areas of the brain are also affected; therefore, investigation into regional changes of bile acid concentrations during $\mathrm{HE}$ is warranted.

\section{Strategies to Deplete or Alter the Composition of the Bile Acid Pool Can Alter the Pathogenesis of HE}

Strategies used to deplete the circulating bile acid levels, such as the use of the bile acid sequestrant cholestyramine, have previously been effective in the treatment of some of the peripheral adverse effects of cholestatic liver damage, such as pruritus. ${ }^{49,50}$ In contrast to this beneficial effect, cholestyramine feeding in acetaminophen-treated mice actually leads to worse liver damage and increased necrosis. ${ }^{51}$ However, to date, no information exists regarding the beneficial effects of cholestyramine treatment in the neurological consequences of liver damage. The data presented herein suggest that reduction of serum and brain bile acids by short-term cholestyramine feeding conferred no reduction in AOM-induced liver damage, but significantly delayed the resulting neurological decline. These findings indicate a novel role for bile acids in the pathophysiological processes associated with the development of HE.

In addition to modulating the total levels of bile acids in the serum, the data presented herein indicate that the composition of the bile acid pool may also be important in determining the susceptibility of mice to the neurological complications of acute liver failure. For example, mice lacking the expression of Cyp7A1, a bile acid synthesis enzyme found predominantly in the liver, but not the brain, of mice, were relatively protected from developing the $\mathrm{HE}$ after AOM injection. As mentioned above, these mice had a reduced total bile acid pool and fecal bile acid content, but a significant reduction in certain bile acids, such as DCA, lithocholic acid, and $\beta$-muricholic acid. ${ }^{16}$ Furthermore, our data suggest that feeding mice with diets enriched with either cholic acid or DCA increased the susceptibility of these mice to the development of HE. DCA-fed mice that were administered AOM had worse neurological decline but exhibited less cerebral edema compared with mice administered AOM and were fed a control diet. Therefore, DCA treatment may be generating an unknown metabolic or cell-signaling consequence that was not identified in these studies and is the subject of further studies in our laboratory. Previous studies using similar concentrations of bile acid-enriched diets suggest that the bioconversion of the enriched bile acid after ingestion may play a large role in the phenomena observed herein. ${ }^{23}$ For example, feeding mice a diet enriched with $1 \%$ cholic acid significantly increased the concentration of DCA, taurocholic acid, and taurodeoxycholic acid. ${ }^{23}$ Similarly, a diet enriched with $0.3 \%$ DCA predominantly increased cholic acid and the taurine conjugates of both species. ${ }^{52}$ Therefore, it is reasonable to assume that the effects of cholic acid or DCA feeding on the neurological decline because of acute liver failure may not solely be because of the 
individual enriched bile acid, but may be because of a metabolite or conjugated version of the bile acid.

\section{The Mechanism of Action of Bile Acids in the Brain Involves ASBT-Dependent Uptake and Activation of FXR}

Circulating bile acids can gain access to the brain via two known mechanisms. First, bile acids can be transported across the BBB through the bile acid transporters found in the choroid plexus. ${ }^{52}$ Second, another route of entry is via the diffusion of bile acids through an increasingly permeable BBB. We, and others, have demonstrated that the BBB is impaired in models of acute and chronic liver damage. ${ }^{26,37,53-56}$ Furthermore, we have recently demonstrated that the circulating bile acids themselves can induce permeability in the BBB via a mechanism involving the Rac1-dependent phosphorylation of the tight junction-associated protein Occludin $1 .{ }^{26}$ The exact mechanisms that lead to bile acid entry into the brain during HE, whether through alterations in bile acid transporters in the choroid plexus or increased passive diffusion through a leaky BBB, are unclear and are areas of ongoing research in our laboratory.

Bile acids are absorbed into the cell via either passive diffusion or active transport through transporters such as ASBT, depending on the polarity of the individual bile acids. Once inside the cell, bile acids can bind to and activate the nuclear receptor FXR. To our knowledge, the current study is the first study to identify the expression of ASBT and FXR in the brain, specifically the frontal cortex. Both ASBT and FXR immunoreactivity was found to colocalize with neuronal markers, suggesting that bile acid signaling may be prevalent in neurons of the frontal cortex. In addition, our data demonstrate an induction in ASBT, FXR, and SHP expression at early stages of neurological decline after AOM injection, followed by a rapid decline once the mice reached hepatic coma, and that blocking FXR translation using Vivo-morpholino technology delays neurological decline. Although FXR signaling has not previously been demonstrated in neurons, the expression of the cell surface bile acid receptor TGR5 has been demonstrated in neurons and astrocytes, ${ }^{57}$ suggesting that machinery for transducing bile acid signaling is present in the brain. Furthermore, the expression of TGR5 has been shown to be down-regulated in a model of ammonia intoxication. ${ }^{57}$ Therefore, it is conceivable that FXR and ASBT may also be present in the brain because our data suggest that the down-regulation of FXR and ASBT expression at the time of hepatic coma may be as a result of a buildup in ammonia in the brain, reported to occur only at late stages of neurological decline after AOM injection. ${ }^{42,58}$ The mechanism by which ASBT/FXR expression is up-regulated at early stages of $\mathrm{HE}$ is unknown, and the downstream consequences of FXR signaling that leads to neurological impairment during acute liver failure warrant further investigation.

\section{Conclusions}

The data presented herein suggest that after liver failure, bile acids in the serum are gaining access to the brain and contributing to the neurological decline associated with HE via a mechanism involving FXR activation. Our results suggest that strategies to decrease serum bile acids or to modulate the bile acid components of the bile acid pool may be a potential therapeutic option, or a supplemental therapy to existing treatments, for the management of $\mathrm{HE}$ because of acute liver failure. Furthermore, modulating FXR signaling in the brain may also be a viable target for the development of treatment paradigms for patients with HE.

\section{Acknowledgments}

We thank Cheryl Galindo, Hae Yong Pae, and Amber Jacobs for technical assistance on this project, Dr. Paul Dawson (Wake Forest University Health Sciences, Winston Salem, NC) for ASBT, Dr. Sandra Erickson (University of California, San Francisco, CA) for the breeding pairs of Cyp7A1 heterozygous knockout mice, and the Central Texas Veterans Health Care System (Temple, TX) for providing resources and use of facilities.

\section{Supplemental Data}

Supplemental material for this article can be found at http://dx.doi.org/10.1016/j.ajpath.2015.10.005.

\section{References}

1. Butterworth RF: Hepatic encephalopathy: a central neuroinflammatory disorder? Hepatology 2011, 53:1372-1376

2. Hazell AS, Butterworth RF: Hepatic encephalopathy: an update of pathophysiologic mechanisms. Proc Soc Exp Biol Med 1999, 222: 99-112

3. Kramer L, Kodras K: Detoxification as a treatment goal in hepatic failure. Liver Int 2011, 31 Suppl 3:1-4

4. Ostapowicz G, Fontana RJ, Schiodt FV, Larson A, Davern TJ, Han SH, McCashland TM, Shakil AO, Hay JE, Hynan L, Crippin JS, Blei AT, Samuel G, Reisch J, Lee WM: Results of a prospective study of acute liver failure at 17 tertiary care centers in the United States. Ann Intern Med 2002, 137:947-954

5. St-Pierre MV, Kullak-Ublick GA, Hagenbuch B, Meier PJ: Transport of bile acids in hepatic and non-hepatic tissues. J Exp Biol 2001, 204: 1673-1686

6. Dawson PA: Role of the intestinal bile acid transporters in bile acid and drug disposition. Handb Exp Pharmacol 2011, 201:169-203

7. Redinger RN: The role of the enterohepatic circulation of bile salts and nuclear hormone receptors in the regulation of cholesterol homeostasis: bile salts as ligands for nuclear hormone receptors. Can J Gastroenterol 2003, 17:265-271

8. Rizzo G, Renga B, Mencarelli A, Pellicciari R, Fiorucci S: Role of FXR in regulating bile acid homeostasis and relevance for human diseases. Curr Drug Targets Immune Endocr Metabol Disord 2005, 5: 289-303

9. Greim H, Trulzsch D, Czygan P, Rudick J, Hutterer F, Schaffner F, Popper H: Mechanism of cholestasis, 6: bile acids in human livers 
with or without biliary obstruction. Gastroenterology 1972, 63: $846-850$

10. Greim H, Trulzsch D, Roboz J, Dressler K, Czygan P, Hutterer F, Schaffner F, Popper H: Mechanism of cholestasis, 5: bile acids in normal rat livers and in those after bile duct ligation. Gastroenterology 1972, 63:837-845

11. Muriel P, Castro V: Dose-response studies of interferon-alpha $2 \mathrm{~b}$ on liver fibrosis and cholestasis induced by biliary obstruction in rats Pharmacology 1997, 54:179-185

12. Raiford DS: Pruritus of chronic cholestasis. QJM 1995, 88:603-607

13. Ma Z, Lee SS, Meddings JB: Effects of altered cardiac membrane fluidity on beta-adrenergic receptor signalling in rats with cirrhotic cardiomyopathy. J Hepatol 1997, 26:904-912

14. Bomzon A, Ljubuncic P: Bile acids as endogenous vasodilators? Biochem Pharmacol 1995, 49:581-589

15. Shneider BL, Dawson PA, Christie DM, Hardikar W, Wong MH, Suchy FJ: Cloning and molecular characterization of the ontogeny of a rat ileal sodium-dependent bile acid transporter. J Clin Invest 1995, 95:745-754

16. Erickson SK, Lear SR, Deane S, Dubrac S, Huling SL, Nguyen L, Bollineni JS, Shefer S, Hyogo H, Cohen DE, Shneider B, Sehayek E, Ananthanarayanan M, Balasubramaniyan N, Suchy FJ, Batta AK, Salen G: Hypercholesterolemia and changes in lipid and bile acid metabolism in male and female cyp7A1-deficient mice. J Lipid Res 2003, 44:1001-1009

17. McMillin M, Galindo C, Pae HY, Frampton G, Di Patre P, Quinn M, Whittington E, DeMorrow S: Gli1 activation and protection against hepatic encephalopathy is suppressed by circulating transforming growth factor $\beta 1$ in mice. J Hepatol 2014, 61:1260-1266

18. McMillin M, Frampton G, Thompson M, Galindo C, Standeford H, Whittington E, Alpini G, DeMorrow S: Neuronal CCL2 is upregulated during hepatic encephalopathy and contributes to microglia activation and neurological decline. J Neuroinflammation 2014, 11 121

19. McMillin M, Frampton G, Tobin R, Dusio G, Smith J, Shin H, Newell-Rogers MK, Grant S, DeMorrow S: TGR5 signaling reduces neuroinflammation during hepatic encephalopathy. J Neurochem 2015, 135:565-576

20. Baskaya MK, Dogan A, Rao AM, Dempsey RJ: Neuroprotective effects of citicoline on brain edema and blood-brain barrier breakdown after traumatic brain injury. J Neurosurg 2000, 92 $448-452$

21. Cao C, Yu X, Liao Z, Zhu N, Huo H, Wang M, Ji G, She H, Luo Z, Yue S: Hypertonic saline reduces lipopolysaccharide-induced mouse brain edema through inhibiting aquaporin 4 expression. Crit Care 2012, 16:R186

22. Bemeur C, Vaquero J, Desjardins P, Butterworth RF: N-acetylcysteine attenuates cerebral complications of non-acetaminopheninduced acute liver failure in mice: antioxidant and anti-inflammatory mechanisms. Metab Brain Dis 2010, 25:241-249

23. Zhang Y, Klaassen CD: Effects of feeding bile acids and a bile acid sequestrant on hepatic bile acid composition in mice. J Lipid Res 2010, 51:3230-3242

24. Mills CO, Rahman K, Coleman R, Elias E: Cholyl-lysylfluorescein: synthesis, biliary excretion in vivo and during single-pass perfusion of isolated perfused rat liver. Biochim Biophys Acta 1991, 1115: $151-156$

25. de Waart DR, Hausler S, Vlaming ML, Kunne C, Hanggi E, Gruss HJ, Oude Elferink RP, Stieger B: Hepatic transport mechanisms of cholyl-L-lysyl-fluorescein. J Pharmacol Exp Ther 2010, 334: 78-86

26. Quinn M, McMillin M, Galindo C, Frampton G, Pae HY, DeMorrow S: Bile acids permeabilize the blood brain barrier after bile duct ligation in rats via Rac1-dependent mechanisms. Dig Liver Dis $2014,46: 527-534$

27. Frampton G, Coufal M, Li H, Ramirez J, DeMorrow S: Opposing actions of endocannabinoids on cholangiocarcinoma growth is via the differential activation of Notch signaling. Exp Cell Res 2010, 316: $1465-1478$

28. DeMorrow S, Glaser S, Francis H, Venter J, Vaculin B, Vaculin S, Alpini G: Opposing actions of endocannabinoids on cholangiocarcinoma growth: recruitment of Fas and Fas ligand to lipid rafts. J Biol Chem 2007, 282:13098-13113

29. Goodenough S, Davidson M, Chen W, Beckmann A, Pujic Z, Otsuki M, Matsumoto I, Wilce P: Immediate early gene expression and delayed cell death in limbic areas of the rat brain after kainic acid treatment and recovery in the cold. Exp Neurol 1997, 145:451-461

30. Frampton G, Invernizzi P, Bernuzzi F, Pae HY, Quinn M, Horvat D, Galindo C, Huang L, McMillin M, Cooper B, Rimassa L, DeMorrow S: Interleukin-6-driven progranulin expression increases cholangiocarcinoma growth by an Akt-dependent mechanism. Gut 2012, 61:268-277

31. DeMorrow S, Francis H, Gaudio E, Venter J, Franchitto A, Kopriva S, Onori P, Mancinelli R, Frampton G, Coufal M, Mitchell B, Vaculin B, Alpini G: The endocannabinoid anandamide inhibits cholangiocarcinoma growth via activation of the noncanonical Wnt signaling pathway. Am J Physiol Gastrointest Liver Physiol 2008, 295:G1150-G1158

32. Livak KJ, Schmittgen TD: Analysis of relative gene expression data using real-time quantitative PCR and the 2(-Delta Delta $\mathrm{C}(\mathrm{T})$ ) Method. Methods 2001, 25:402-408

33. Goodenough S, Davidson M, Kidd G, Matsumoto I, Wilce P: Cell death and immunohistochemistry of p53, c-Fos and c-Jun after spermine injection into the rat striatum. Exp Brain Res 2000, 131: $126-134$

34. Cash WJ, McConville P, McDermott E, McCormick PA, Callender ME, McDougall NI: Current concepts in the assessment and treatment of hepatic encephalopathy. QJM 2010, 103:9-16

35. Hori T, Chen F, Baine AM, Gardner LB, Nguyen JH: Fulminant liver failure model with hepatic encephalopathy in the mouse. Ann Gastroenterol 2011, 24:294-306

36. Jayakumar AR, Ruiz-Cordero R, Tong XY, Norenberg MD: Brain edema in acute liver failure: role of neurosteroids. Arch Biochem Biophys 2013, 536:171-175

37. McMillin MA, Frampton GA, Seiwell AP, Patel NS, Jacobs AN, DeMorrow S: TGFbetal exacerbates blood-brain barrier permeability in a mouse model of hepatic encephalopathy via upregulation of MMP9 and downregulation of claudin-5. Lab Invest 2015, 95:903-913

38. Nguyen JH, Yamamoto S, Steers J, Sevlever D, Lin W, Shimojima N, Castanedes-Casey M, Genco P, Golde T, Richelson E, Dickson D, McKinney M, Eckman CB: Matrix metalloproteinase-9 contributes to brain extravasation and edema in fulminant hepatic failure mice. J Hepatol 2006, 44:1105-1114

39. Chastre A, Belanger M, Nguyen BN, Butterworth RF: Lipopolysaccharide precipitates hepatic encephalopathy and increases blood-brain barrier permeability in mice with acute liver failure. Liver Int 2014 34:353-361

40. Butterworth RF: The liver-brain axis in liver failure: neuroinflammation and encephalopathy. Nat Rev Gastroenterol Hepatol 2013, 10:522-528

41. Bemeur C, Qu H, Desjardins P, Butterworth RF: IL-1 or TNF receptor gene deletion delays onset of encephalopathy and attenuates brain edema in experimental acute liver failure. Neurochem Int 2010, $56: 213-215$

42. Matkowskyj KA, Marrero JA, Carroll RE, Danilkovich AV, Green RM, Benya RV: Azoxymethane-induced fulminant hepatic failure in C57BL/6J mice: characterization of a new animal model. Am J Physiol 1999, 277:G455-G462

43. Kakiyama G, Pandak WM, Gillevet PM, Hylemon PB, Heuman DM, Daita K, Takei H, Muto A, Nittono H, Ridlon JM, White MB, Noble NA, Monteith P, Fuchs M, Thacker LR, Sikaroodi M, Bajaj JS: Modulation of the fecal bile acid profile by gut microbiota in cirrhosis. J Hepatol 2013, 58:949-955 
44. Bron B, Waldram R, Silk DB, Williams R: Serum, cerebrospinal fluid, and brain levels of bile acids in patients with fulminant hepatic failure. Gut 1977, 18:692-696

45. Tripodi V, Contin M, Fernandez MA, Lemberg A: Bile acids content in brain of common duct ligated rats. Ann Hepatol 2012, 11:930-934

46. Choi JM, Kim YH, Roh SY: Acute hepatic encephalopathy presenting as cortical laminar necrosis: case report. Korean J Radiol 2013, 14:324-328

47. Arnold SM, Els T, Spreer J, Schumacher M: Acute hepatic encephalopathy with diffuse cortical lesions. Neuroradiology 2001, 43: $551-554$

48. Matsusue E, Kinoshita T, Ohama E, Ogawa T: Cerebral cortical and white matter lesions in chronic hepatic encephalopathy: MRpathologic correlations. AJNR Am J Neuroradiol 2005, 26: $347-351$

49. Kremer AE, Beuers U, Oude-Elferink RP, Pusl T: Pathogenesis and treatment of pruritus in cholestasis. Drugs 2008, 68:2163-2182

50. Scaldaferri F, Pizzoferrato M, Ponziani FR, Gasbarrini G, Gasbarrini A: Use and indications of cholestyramine and bile acid sequestrants. Intern Emerg Med 2013, 8:205-210

51. Bhushan B, Borude P, Edwards G, Walesky C, Cleveland J, Li F, Ma X, Apte U: Role of bile acids in liver injury and regeneration following acetaminophen overdose. Am J Pathol 2013, 183: $1518-1526$

52. Choudhuri S, Cherrington NJ, Li N, Klaassen CD: Constitutive expression of various xenobiotic and endobiotic transporter mRNAs in the choroid plexus of rats. Drug Metab Dispos 2003, 31: $1337-1345$

53. Chen F, Ohashi N, Li W, Eckman C, Nguyen JH: Disruptions of occludin and claudin-5 in brain endothelial cells in vitro and in brains of mice with acute liver failure. Hepatology 2009, 50:1914-1923

54. Faropoulos K, Chroni E, Assimakopoulos SF, Mavrakis A, Stamatopoulou V, Toumpeki C, Drainas D, Grintzalis K, Papapostolou I, Georgiou CD, Konstantinou D: Altered occludin expression in brain capillaries induced by obstructive jaundice in rats. Brain Res 2010, 1325:121-127

55. Shimojima N, Eckman CB, McKinney M, Sevlever D, Yamamoto S, Lin W, Dickson DW, Nguyen JH: Altered expression of zonula occludens-2 precedes increased blood-brain barrier permeability in a murine model of fulminant hepatic failure. J Invest Surg 2008, 21: $101-108$

56. Wright G, Davies NA, Shawcross DL, Hodges SJ, Zwingmann C, Brooks HF, Mani AR, Harry D, Stadlbauer V, Zou Z, Williams R, Davies C, Moore KP, Jalan R: Endotoxemia produces coma and brain swelling in bile duct ligated rats. Hepatology 2007, 45: $1517-1526$

57. Keitel V, Gorg B, Bidmon HJ, Zemtsova I, Spomer L, Zilles K, Haussinger D: The bile acid receptor TGR5 (Gpbar-1) acts as a neurosteroid receptor in brain. Glia 2010, 58:1794-1805

58. Belanger M, Cote J, Butterworth RF: Neurobiological characterization of an azoxymethane mouse model of acute liver failure. Neurochem Int 2006, 48:434-440 